\title{
Capacidades de Tecnologías de Información y Capacidades de Negocio Electrónico (E-Business): Efecto Mediador de la Capacidad de Absorción
}

\author{
Andrés A. García ${ }^{(1)}$, Henry E. Díaz ${ }^{(2)}$ y José E. Arias-Pérez ${ }^{(1)}$ \\ (1) Universidad de Antioquia, calle 70 No. 52-21 oficina 13-105, Medellín, Colombia \\ (e-mail: aaugusto.garcia@udea.edu.co; jenrique.arias@udea.edu.co) \\ (2) Universidad de San Buenaventura, carrera 56C No. 51-110, Medellín, Colombia \\ (e-mail: diazhenrysalud@gmail.com)
}

Recibido Jun. 17, 2016; Aceptado Ago. 29, 2016; Versión final Sep. 29, 2016, Publicado Feb. 2017

\begin{abstract}
Resumen
El objetivo del artículo es establecer el efecto mediador de la capacidad de absorción en la relación entre capacidades de tecnologías de información (CTI) y las capacidades de negocios electrónicos o E-Business (CEB) en una muestra de empresas que pertenecen a sectores intensivos en el uso de tecnologías de información. Se utilizaron ecuaciones estructurales por el método de mínimos cuadrados parciales, análisis bootstrap-percentil y test de la varianza explicada para comprobar la existencia y la magnitud del efecto mediador. Se encontró que existe una mediación parcial y por lo tanto la incidencia de las CTI sobre las CEB depende parcialmente del desarrollo de habilidades organizacionales blandas orientadas a la creación de valor a partir de la adquisición y asimilación de conocimiento externo. Se concluye que los recursos tecnológicos son de gran importancia pero insuficientes para garantizar el éxito de la operación digital del negocio.
\end{abstract}

Palabras clave: tecnologías de información; e-business; gestión del conocimiento; gestión de tecnología

\section{Information Technologies and E-business Capabilities: The Absorptive Capacity mediating effect}

\begin{abstract}
The objective of this article is to establish the mediating effect of the absorptive capacity in the relationship between information technology capabilities (ITC) and E-Business capabilities (EBC) in a sample of firms belonging to sectors of intensive use of information technology. Structural equations are used including the partial least square method, the bootstrap-percentile analysis and the explained variance test in order to check the existence and magnitude of the mediating effect. It was found that there is a partial mediation and therefore the impact of ICT on the CEB depends partly on the development of soft organizational skills aimed at creating value from the acquisition and assimilation of external knowledge. It is concluded that technological resources are of great importance but insufficient to ensure the success of digital business operation.
\end{abstract}

Keywords: information technology; e-business; knowledge management; technology management 


\section{INTRODUCCIÓN}

El Foro Económico Mundial (2015) indica que el potencial transformador de las tecnologías de información y comunicación (TIC), en los últimos 13 años, ha sido un mecanismo de apalancamiento para mejorar la calidad de vida de las personas, fomentar el crecimiento económico y crear oportunidades para los individuos, las empresas y naciones de todo el mundo. Los indicadores oficiales respecto al sector TIC en Colombia para el año 2014, mostraron un crecimiento del 3,9\% (frente a un 2,7\% del 2013) y una representación del 3,13\% en el PIB del país en el 2014. Estos datos se miden en el PIB desde el punto de vista de la oferta, sumando los valores agregados que añaden las empresas de diferentes etapas de producción en el sector de TIC (MinTIC, 2015). Estas cifras dejan posicionado al sector como uno de alto impacto en la economía nacional. En un contexto global esbozado por el índice de disponibilidad de la red (Networked Readiness Index, NRI), del Foro Económico Mundial, Colombia se ubica en el ranking 89 en lo relacionado con Absorción Tecnológica, puesto 67 en el uso de Internet Business to Business (B2B), y 62 en el uso de Internet Business to Consumer (B2C), dentro de una lista de 143 países desarrollados y emergentes (FEC, 2015). Este panorama muestra que aunque en Colombia se está trabajando fuertemente en el sector TIC, aún son necesarios esfuerzos para desarrollar capacidades que permitan obtener más valor de estas tecnologías para estar en un nivel cercano de la élite mundial.

A medida que estos esfuerzos en el desarrollo de las TIC aumentan, también lo hace la necesidad por definir y medir las capacidades de las Tecnologías de la Información (CTI). La literatura existente en este tema es extensa y básicamente se enfoca en entender qué son las Capacidades de TI, cómo medir su impacto en las organizaciones y sus diferentes procesos, qué clases de competencias y capacidades se desarrollan a su alrededor, y qué tan rentables son las inversiones relacionadas con TI. Una empresa eficiente en el manejo de los recursos relacionados con las CTI, desarrolla la capacidad de adquirir, implementar, combinar y reconfigurar dichos recursos en apoyo y mejora de las estrategias de negocio y procesos de trabajo (Sambamurthy y Zmund, 1997), de manera que se pueda lograr una ventaja competitiva basada en capacidades difícilmente imitables (Bharadwaj, 2000). Una alternativa para la creación de valor y desempeño empresarial a partir de las capacidades de TI, es la generación de las Capacidades de E-Business. (Zhu et al., 2004).

Con base en las CTI, el desarrollo de las Capacidades de E-Business (CEB) permite que procesos organizacionales como la cadena de suministros y los procesos de CRM sean ejecutados de forma más efectiva sobre medios de comunicación digitales (Chi et al., 2010), o procesos de mercadeo, de inteligencia de negocio, comercio y colaboración con socios externos (Raymond y Bergeron, 2008). Las CEB permiten conectar mediante flujos de información, las actividades de la cadena de suministro con la operación digital del negocio (Zhu et al., 2015) usando las conexiones a Internet, para trascender los límites geográficos que enmarcan la relación con los socios de la cadena de suministro, para apoyar las operaciones del negocio de forma digital, incluidas las compras, la gestión de canales y el servicio al cliente.

La relación estrecha entre las CTI y las CEB ha sido clarificada a lo largo de los años. Las CEB requieren unas capacidades en CTI maduras para su adecuado funcionamiento, escalabilidad, crecimiento futuro y demanda de más recursos (Menascé, 2000). Además, es necesario tener la flexibilidad de aplicar las CTI de forma acorde a las exigencias puntuales que las CEB demanden, dependiendo del escenario donde se desplieguen (Weill y Vitale, 2002). También es sabido que una base fundamental de las CEB es la infraestructura que prestan las CTI, y en este sentido, a mayor desarrollo de CTI mayor será el de CEB (Koellinger, 2008; Barney y Ray, 2015; Singh y Teng, 2016). En Colombia, la discusión entre CTI y CEB ha sido prácticamente inexistente. En lo relacionado al tema, en el país se ha investigado el impacto de las TIC en el desempeño empresarial (Alderete y Gutiérrez, 2012) y el innovador (Gálvez, 2014), con resultados que indican una influencia positiva.

De otra parte, en la actualidad se ha comenzado a demostrar que los efectos de las CTI sobre los distintos constructos organizacionales están mediados por las rutinas organizacionales que permiten adquirir conocimiento externo, asimilarlo, transformarlo y explotarlo. Estas rutinas organizacionales, que conforman la capacidad de poder procesar el conocimiento externo son conocidas como la Capacidad de Absorción (CA), la cual se define como la acción de reconocer el valor de nueva información, su asimilación y aplicación para fines comerciales (Cohen y Levinthal, 1990) y se considera como un constructo dinámico, es decir, que impulsa a las organizaciones hacia la evolución (Zahra y George, 2002; Patterson y Ambrosini, 2016).

Por ende, la CA puede complementar el desarrollo de múltiples capacidades, puesto que es el instrumento para que las organizaciones exploren el entorno y extraigan el conocimiento que lleva a mejorar los diferentes indicadores de sus negocios, conduciendo así a la creación de más valor. Esta afirmación es particularmente importante en el efecto de complementariedad que la CA ejerce sobre las relaciones de 
influencia que tienen las CTI sobre diferentes constructos organizacionales. En ese sentido, se ha demostrado la influencia mediadora de la CA en diferentes situaciones en las que las CTI tienen relación con ciertas capacidades organizacionales, el desempeño empresarial y el desarrollo de nuevos productos (Liu et al., 2013; Addas y Pinsonneault, 2014). Por ejemplo, se han encontrado estudios que han demostrado el efecto mediador de la CA en la relación entre las Capacidades de TI y la agilidad organizacional (Roldán et al., 2015). También, la relación entre los conocimientos basados en CTI y la habilidad de comercializar innovaciones está mediada por la CA (Datta, 2012).

Sin embargo, dentro del universo de investigaciones al respecto, es notoria la ausencia de trabajos donde se analice el efecto de la mediación de la CA en la relación entre CTI y las CEB, la cual tendría todo el sentido porque en estudios previos se ha discutido ampliamente que las CTI y las CEB tienen que estar soportadas por conocimientos y rutinas que van más allá de lo técnico y necesitan ser complementadas por una variedad de aportes con matices de múltiples disciplinas (Maditinos et al., 2014). Por lo tanto, el presente artículo pretende llenar el vacío existente en la literatura, estableciendo la existencia de la mediación de CA en la relación entre CTI y CEB en empresas intensivas en el uso de TI.

\section{ANTECEDENTES}

En la literatura académica se ha definido a las CTI como "la habilidad de un sistema de cómputo (EI conjunto de computadoras y tecnologías relacionadas en una organización) para almacenar, procesar, y comunicar información" (Nakata et al., 2008). En los primeros estudios relacionados con el tema, esté concepto estuvo limitado sólo al aspecto netamente técnico e instrumentalista, pero han surgido corrientes que han ampliado la definición de este constructo, y le han adjudicado características que incluyen más dimensiones. Es así como en la actualidad, las CTI son consideradas de una forma más amplia, que considera los aspectos técnicos, incluye también procesos, recursos humanos y hasta estrategias.

\section{Capacidades de tecnologías de la información (CTI)}

Algunos autores han demostrado que la inversión en TI es fácilmente imitada por los competidores. Estas inversiones por si solas no proveen ventajas sostenidas en el tiempo (Carr, 2003). En cambio, la forma en que las compañías apalancan su inversión para crear recursos y habilidades únicas en TI determinan la efectividad de la compañía (Bharadwaj, 2000), en consecuencia, los resultados de una firma pueden depender en gran medida, del grado de desarrollo de CTI.

En este sentido, una definición más amplia divide las CTI en dimensiones: rutinas y arquitecturas de TI, infraestructura de TI, recursos humanos de TI y activos relacionales de TI (Jiao et al., 2008). También, otra visión atribuye a las CTI los componentes relacionados con la infraestructura de TI, asociación del negocio de $\mathrm{TI}$, pensamiento estratégico de negocios en $\mathrm{TI}$, integración de procesos de negocios en $\mathrm{TI}$, administración de TI y enlaces externos (Chen, et al., 2014). Para el caso de este estudio las CTI tienen como fundamento tres dimensiones: capacidad de infraestructura de TI (fundamentación tecnológica), capacidad de extensión de las TI sobre el negocio (pensamiento estratégico) y la postura proactiva de TI (orientación hacia las nuevas oportunidades) (Lu y Ramamurthy, 2011). Esta definición va más allá de la simple categorización de las CTI con constructos que aportan al direccionamiento de la empresa incluyendo un componente estratégico que implica análisis, conocimiento y talento y otro componente proactivo que involucra posturas de vigilancia del entorno, innovación y mercados. Así, esta concepción incluye el factor humano, el conocimiento técnico y los procesos empresariales.

\section{Capacidades de los negocios electrónicos o e-business (CEB)}

Las plataformas de E-business, más allá de ejecutar una serie de actividades que simplemente comprenden transacciones comerciales a través de internet, se soportan en el uso estratégico de las TI para lograr mayor efectividad en las relaciones con las distintas entidades y personas que componen la cadena de suministros (Zhu K., 2004). En este sentido, el E-business es un mecanismo que impulsa la transformación de procesos claves, reduciendo costos e incrementando ventas simultáneamente (Kaefer y Bendoly, 2004). Además, el e-business provee un soporte eficiente para compartir información y conocimiento e integrar los procesos de negocios entre los socios de la cadena de suministros (Saraf et al., 2007; Singh y Teng, 2016).

A pesar de esto, algunas empresas que han hecho inversiones en tecnologías relacionadas con Internet no han percibido el retorno esperado de negocios operados sobre e-business (Zhu y Kraemer, 2005). Este problema evidencia que los e-business son difíciles de administrar y los fracasos al respecto son abundantes (Poon y Wagner, 2001; Saraf et al., 2007). Para evitar esta situación problemática, se ha empezado a reconocer la importancia del desarrollo de las capacidades que permitan desplegar y explotar los recursos de e-business para la creación de ventajas competitivas. Dichas capacidades son las Capacidades de E-business (CEB) (Zhu K., 2004; Barua et al., 2004; Eikebrokk y Olsen, 2007; Matidinos et al., 2014). 
Apoyando lo anterior y para efectos de esta investigación, las CEB son definidas como las habilidades organizacionales que permiten conectar mediante flujos de información, las actividades de la cadena de suministro con la operación digital del negocio (Zhao et al., 2008; Chi et al., 2010; Zhu et al., 2015). En la literatura, algunos autores han señalado que las CEB abarcan procesos como las relaciones Inter-Intra EBusiness, (Wu, et al., 2011; Shi y Liao, 2015), capacidades de comunicación y de Inteligencia de negocios (Raymond y Bergeron, 2008), capacidades de Colaboración (Sanders, 2007; Jiang y Zhao, 2010) y capacidad de compra en línea (Mishra et al., 2007; Chang et al., 2013).

En este estudio se adopta un modelo de las CEB que comprende tres dimensiones: la capacidad de Compras en Línea o e-procurement, la capacidad de Gestión de Canales en Línea y la capacidad de Servicio al cliente en Línea (Johnson y Whang, 2002; Zhu et al., 2015). Estas dimensiones describen, en un modelo simple, todas las relaciones creadas a raíz del uso de las plataformas de e-business: flujos de información y relaciones con clientes y proveedores.

Ampliando la definición de estos conceptos, se asevera que la capacidad de compras en línea se concibe como la habilidad de operar plataformas sobre Internet para coordinar las actividades de compras, negociaciones y transacciones, así como la programación y gestión de la demanda de materias primas e insumos (Mishra et al., 2007; Chang et al., 2013). La capacidad de gestión de canales en línea se precisa como la habilidad de operar plataformas sobre Internet para dirigir la gestión de canales, realizar promociones, lanzamientos de productos, fijación de precios y el cumplimiento de órdenes (Saraf et al., 2007; Oh et al., 2008; Singh y Teng, 2016) y la capacidad de servicio al cliente en línea, se define como la habilidad de operar plataformas sobre Internet para dirigir los servicios que hacen seguimiento a las demandas de los clientes actuales y potenciales, la gestión de sus relaciones y el acompañamiento de sus procesos en los diferentes canales de atención de la compañía. (Eng, 2008; Hung et al., 2014).

\section{CTI y $C E B$}

Los resultados de cada una de estas dimensiones de la CEB, están directamente relacionadas con el grado de desarrollo de las CTI. Las CTI proveen instrumentos para que las actividades clave relacionadas con el despliegue y el uso de sistemas E-business se desempeñen de una forma eficiente y efectiva mediante la proporción de sistemas de infraestructura, planeación y estrategias relacionadas con TI, como la creación de políticas y cambios culturales para la adopción y el uso de las tecnologías (Wu et al., 2011).

De forma notable, la capacidad de infraestructura de TI se utiliza en todas las iniciativas de las CEB al conectar las diferentes partes de la empresa con los proveedores, clientes y aliados estratégicos haciendo uso de Internet (Yeh et al., 2014). Esta conexión es posible debido a que la capacidad de Infraestructura de TI ofrece a los usuarios los medios fundamentales para la prestación de servicios de comunicaciones y administración de la información (computadores, dispositivos, servidores, software y hardware) que se acoplan a las necesidades de las empresas en los procesos de negociación (Cano y Baena, 2015).

Pero la relación de las CTI con las CEB no sólo se limita a lo técnico e instrumental, la influencia de las dimensiones de las CTI es considerable en varios aspectos de las CEB. Esta afirmación es particularmente importante en la colaboración que debe existir entre los socios que participan en la operación de las CEB. El uso eficiente de las TI depende del grado de cooperación entre los socios externos e internos a la organización (Oh et al., 2014). Para crear lazos de colaboración con socios externos, que serán usuarios de plataformas de e-business, es necesario contar con CTI maduras que permitan la creación de sistemas de TI compatibles y amigables con los sistemas de las empresas socias, es decir, se debe contar con un sentido proactivo de TI que permita observar la conveniencia de la tecnología que se implementa, un sentido táctico de las TI que permita crear lazos fuertes y dinámica de negocios y una infraestructura de TI robusta que soporte el flujo de datos que soportae las transacciones en línea (Barua et al., 2004).

Otra situación concreta, se da en la forma en las que las CTI impactan el proceso de compras en línea, permitiendo la automatización de flujos de trabajo, regulando la capacidad de gasto de las organizaciones e identificando nuevas oportunidades de compra a través de Internet. (Davila et al., 2003). Además, permite la reducción de costos, acortando el tiempo para diligenciamiento de órdenes, bajando el costo de inventarios y el precio pagado por bienes y servicios, y prepara a la organización para incrementar la colaboración y planeación tecnológica con socios de negocios (Croom, 2000; Roche, 2001).

Por otra parte, para responder de manera rápida y eficiente a los requerimientos del mercado, es necesaria una gestión de canales a través de herramientas de e-business que recoja información de todos los actores involucrados en la cadena de suministros, es decir, desde productores y proveedores hasta los clientes, que permitirá entender los cambios en la demanda y de la competencia y en general del mercado y sus requerimientos. Toda esta información circula en un flujo constante que necesita de tecnología de TI adecuada para seleccionar y administrar los datos relevantes que conlleven a un análisis global de la posición de la compañía y su oferta en el mercado para la toma de decisiones, gestión de cambios y 
reingeniería de procesos. Todo lo anterior está ligado al poder financiero y la efectividad de la gestión estratégica de los colaboradores de la compañía. (Armstrong y Kotler, 2005; Paksoy et al., 2012)

En resumen, la CTI influye sobre la CEB, proveyendo un abanico de opciones tecnológicas para el despliegue de las herramientas electrónicas usadas en la operación de e-business representadas en medios digitales de comunicación, hardware y software y que evolucionan constantemente. Adicionalmente, la conectividad eficiente que proporcionan las $\mathrm{TI}$ son un catalizador de las relaciones y acciones colaborativas entre socios y empresas porque se facilitan los flujos de trabajo y de información para reducir costos y tiempos. Las posturas proactivas de TI permiten vigilar el mercado para adaptar nuevas tecnologías que mejoren el desempeño de las plataformas de E-business y prestar mejores servicios a clientes y proveedores. Basado en lo anterior, este estudio propone la siguiente hipótesis H1: Las CTI tienen un impacto positivo sobre las CEB (ver figura 1)

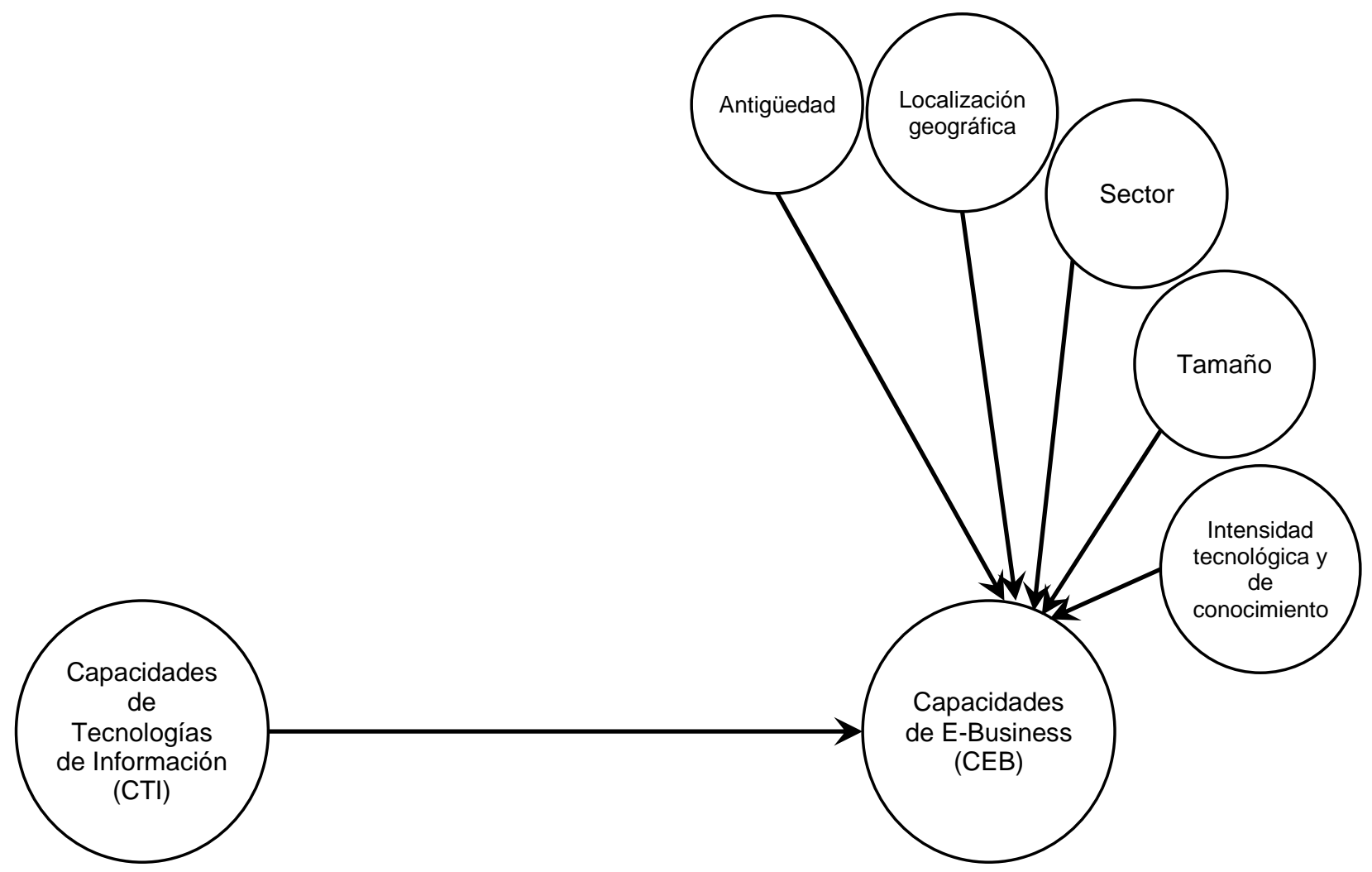

Fig. 1: Modelo directo entre CTI y CEB

\section{Capacidad de absorción (CA)}

Como ya se ha indicado, existe una relación plenamente establecida entre CTI y CEB. Sin embargo, también es sabido que es necesaria la intervención de capacidades adicionales para obtener el éxito en la implementación de procesos corporativos en Internet, que representen un gran valor para las empresas (Zhao et al., 2008). En ese sentido, se ha establecido que la implementación de e-business sufre de algunos inconvenientes, sobre todo en países en vías de desarrollo, básicamente concentrados en problemas regulatorios, de seguridad y de despliegue de infraestructura de TIC (Shakir et al., 2007; Janita y Chong, 2013). De esta forma, se puede observar que el proceso de desarrollo de CEB, está también supeditado a la existencia de rutinas organizacionales que permitan obtener información que se pueda usar para crear valor a partir de la captura de conocimiento externo (Cegarra et al., 2007; Raymond y Bergeron, 2008), lo que corresponde en otras palabras a la Capacidad de Absorción.

De este modo, la capacidad de absorción es definida inicialmente como la habilidad de una empresa de reconocer información nueva y externa, asimilarla y aplicarla a fines comerciales (Cohen y Levinthal, 1990). Extendiendo esta definición inicial, se puede concebir la CA como "la habilidad relativa de una organización para desarrollar un conjunto de rutinas organizacionales y procesos estratégicos con los cuales se adquiere, asimila, transforma y explota el conocimiento adquirido desde el exterior de la organización con el fin de crear valor" (Jiménez et al., 2011).

Acorde con la definición, la literatura señala cuatro dimensiones que componen la CA. La Adquisición de información es entendida como el reconocimiento de nueva información que tiene el potencial de dar valor 
(Cohen y Levinthal, 1990) y como se transfiere el conocimiento de una organización a otra. (Zahra y George, 2002; Patterson y Ambrosini, 2016). En la dimensión de asimilación, la organización entiende con sus propias rutinas el conocimiento externo para obtener ventajas de él. Luego, en la dimensión de transformación, se combina el nuevo conocimiento con el existente y se internaliza creando nuevas estructuras de conocimiento. Por último, en la dimensión de explotación se generan los desarrollos de rutinas para crear nuevos bienes, sistemas y procesos y mejorar o crear competencias (Jiménez et al., 2011).

Una vez entendidas las dimensiones de la $\mathrm{CA}$, se puede analizar de forma más clara como es su dinámica de mediación en la relación entre las CTI y las CEB. Para entender este efecto mediador, se debe dar una mirada a los medios de comunicación, instrumentos y programas informáticos que provee la CTI y que son explotados en los procesos apalancados por las CEB proporcionan métodos de integración y contacto. Pero hay evidencia empírica que declara que las plataformas de E-business son propensas a fracasar (Poon y Wagner, 2001; Zhu y Kraemer, 2005; Wu et al., 2011; Yeh et al., 2014). Si la implementación y operación de las CEB se hace con un enfoque soportado por las habilidades propias de CA, el riesgo de fracaso es menor (Lin y Lee, 2005; Lee et al., 2007). Las CA refuerzan la influencia de las CTI buscando fuentes de información acerca de los mercados, de nuevas tecnologías, de proveedores, logística de abastecimiento y ventas (Sáenz et al., 2014), también permite la transformación de nuevo conocimiento a partir de los conocimientos previos y su aplicación a los servicios de E-business (Liu et al., 2013).

También, el efecto de mediación de la CA en la relación CTI y CEB se presenta en los procesos de compra en línea, dinamizados por cada una de las dimensiones de CTI. No obstante, si un sistema de compras en línea se limita sólo al plano transaccional, las ventajas de crear lazos sólidos con los proveedores, no serán percibidos (Lane y Lubatkin, 1998). Para evitar este inconveniente, es preciso hacer uso de las rutinas de CA, para absorber conocimiento del entorno que permita implementar de forma robusta, segura y amigable, las herramientas que la CTI proporciona a las CEB, y poder así desplegar rutinas de colaboración y retroalimentación, la agilidad en los procesos relacionados con la compra de suministros y el establecimiento de canales de comunicación efectivos con el grupo de proveedores que posibilite el establecimiento de economías de escala eficaces y oportunas, de acuerdo a las necesidades de abastecimiento de la organización, y del mismo modo, permita construir relaciones de confianza con nuevos proveedores que podrán suplir nuevos requerimientos o asegurar los procesos de mejora en el área de compras. (Chang y Gotcher, 2010; Oh et al., 2014).

De forma análoga, la mediación de CA también se evidencia en los procesos de gestión de canales en línea, en los cuales, el flujo de la información respecto a distribuidores, mercadeo, productos y logística es requisito fundamental a cumplir por parte las CTI para su correcto funcionamiento (Dimitriadis y Koh, 2005). Sumado a lo anterior, si se explotan las habilidades representadas por las CA y se incorporan de manera medular en los diferentes procesos de tipo estratégico y de planeación de e-business, las compañías aprovecharán de manera óptima los recursos de CTI, haciendo tratamiento a los flujos de información que cursan sobre los canales en línea, y así podrán absorber el conocimiento externo que permite entender la dinámica actual del mercado y sus tendencias, y transformar y explotar el nuevo conocimiento absorbido para implementar estrategias de precios, posicionamiento de productos, canales de promoción y de distribución en línea (Lane y Lubatkin, 1998; Malhotra et al., 2005). La utilización y apropiación de estos recursos es fundamental para el desarrollo de las CEB, si se quiere crear elementos distintivos y difíciles de imitar por la competencia así como predecir las necesidades de socios y clientes y adelantarse a sus requerimientos por medio de la absorción de conocimiento antes de cambios en el entorno, siendo esto un elemento valioso para la competitividad empresarial y el desempeño de las plataformas de E-business (Cegarra et al., 2007). De esta forma, se pueden crear posiciones dominantes en la forma de gestionar los canales electrónicos. (Williams y Moore, 2007). Así, una firma con CA superior, es mejor percibiendo la información sobre los cambios en el mercado y aprendiendo de las experiencias acerca de la operación de los canales son plataformas de e-business (Liu et al., 2013).

El manejo de la capacidad del servicio al cliente en línea es quizá la porción más sensible de los procesos de CEB, puesto que el servicio al cliente es considerado como uno de los procesos más críticos en las empresas (Lee y Chang, 2007). Por lo anterior, se dispone de Servicios e infraestructura de TI para soportar las diferentes fases que involucran el servicio al cliente (Ray et al., 2005). De este modo, mientras las CTI fortalecen los medios de soporte para el intercambio de información con los clientes sobre plataformas de ebusiness, las habilidades representadas en la CA permiten absorber conocimiento que permita tomar acciones en cuanto a los gustos y características de los clientes que serán la clave de éxito para fortalecer las relaciones comerciales, al diseñar productos y servicios únicos que se adelanten a sus necesidades y las suplan de manera óptima (Chen y Ching, 2005; Raymond et al., 2016). Dicho conocimiento del universo de clientes se logra con un adecuado despliegue de la CA, al estudiar y diseñar estrategias que permitan 
solucionar problemas que preocupan a los clientes como la seguridad de las plataformas de e-business, su costo adicional y los problemas que se dan al acceder a internet (Ericsson, 2013).

En conclusión, aunque se observa una relación fuerte en la forma en la que CTI se relaciona con CEB, ofreciendo herramientas tecnológicas que permiten la gestión de las comunicaciones y datos, la funcionalidad de las plataformas derivadas de esta relación puede no tener la fortaleza necesaria para la demanda del entorno organizacional. Se ha planteado que el conocimiento que se adquiere, asimila, transforma y explota por medio de la CA, permite enriquecer la relación de CTI sobre CEB, creando relaciones más estrechas con proveedores, cuya base fundamental se construye en la confianza y ayuda mutua; la gestión óptima de los canales de distribución y mercadeo, siendo posible atender clientes y distribuidores de formas diversas y efectivas; observando y analizando tendencias y comportamiento del mercado para afianzar políticas de precios, de logística de distribución, promoción y desarrollo de nuevos productos; al mismo tiempo que se dinamiza la relación con clientes actuales y el establecimiento de acercamientos con clientes potenciales, todo ello porque la organización establece mecanismos para conocer las necesidades y percepciones del grupo de clientes y grupos de interés frente a las propias plataformas de e-business, siendo posible actuar incluso anticipadamente ante un cambio abrupto, una nueva tendencia, necesidad de innovación o reingeniería. De acuerdo a lo anterior, se plantea la siguiente hipótesis H2: La relación entre CTI y CEB esta mediada por la CA (ver figura 2)

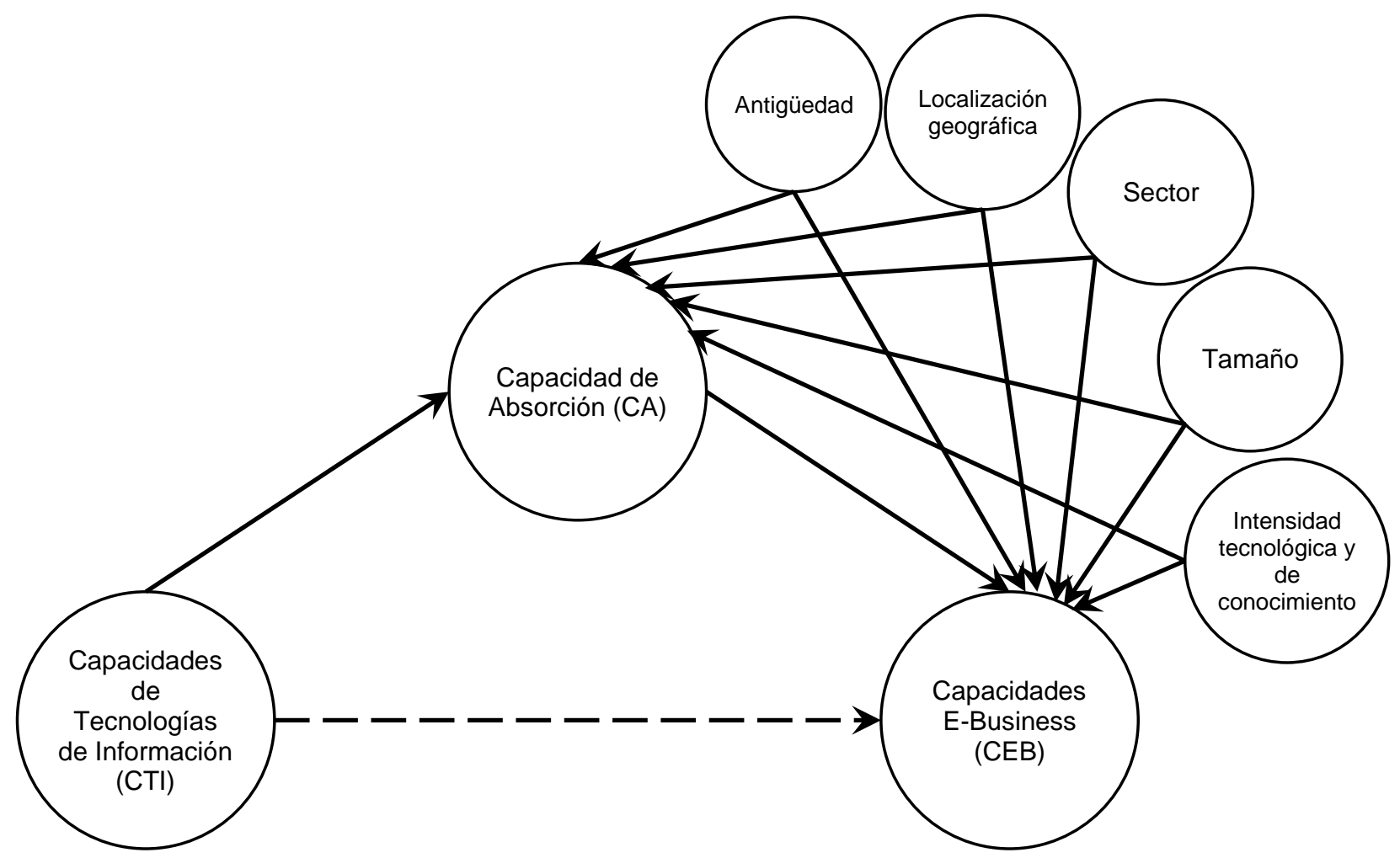

Fig. 2: Modelo mediado de CA en la relación entre CTI y CEB.

\section{METODOLOGÍA}

El modelo de hipótesis de probó en una muestra de empresas manufactureras y de servicios situadas en Colombia, un país emergente y tecnológicamente seguidor, ubicándose en el ranking 89 en lo relacionado con el Nivel de Absorción Tecnológica de las Empresas, puesto 67 en el uso de Internet Business to Business (B2B), y 62 en el uso de Internet Business to Consumer (B2C), dentro de una lista de 143 países (FEC, 2015). Según el Índice de desarrollo de las TIC 2014, Colombia se ubica en el puesto 77, por encima de países de la región como Venezuela puesto 80, Ecuador 88 y Perú 105 (Unidad Internacional de Telecomunicaciones, 2014). De igual modo los indicadores oficiales respecto al sector TIC en Colombia para el año 2014, muestran un crecimiento del 3,9\% frente al 2,7\% del 2013 y una representación del 3,13\% en el PIB del país en el 2014 (Marulanda et al., 2013; MinTIC, 2015; López et al., 2015).

Por consecuencia, las cifras reflejan que Colombia ha atestiguado un progreso importante en el desarrollo de las TIC ayudada por crecimiento económico sano, inversiones en nuevas tecnologías e iniciativas del gobierno para facilitar cobertura de banda ancha en áreas rurales, es uno de los mercados más grandes de las telecomunicaciones de América latina y con fuerte crecimiento. La red de fibra óptica cubre $80.0 \%$ de 
territorio colombiano, 769 municipios, y la cuarta generación de tecnología de datos móviles facilitan el crecimiento gradual de la infraestructura física de las TIC. Asimismo, tiene una de las tasas más altas de ebusiness en América Latina, siendo uno de los líderes regionales del crecimiento de e-business. Por su parte, las empresas colombianas se reconocen por su despliegue en tecnología reflejado en la penetración de la computadora y el internet, utilizados en un $100 \%$ en 2014 , banda ancha en un $90,4 \%$, órdenes en línea en 55,5\%, haciéndose evidente los progresos en servicios en línea (MinTIC, 2015).

La muestra está conformada por empresas manufactureras y de servicios, tanto Pymes como grandes empresas, que pertenecen a sectores intensivos en el uso de TI, por el peso de actividades de negocios electrónicos superiores al $60 \%$ de acuerdo con el Departamento Administrativo Nacional de Estadística (DANE, 2014) (ver Tabla 1). Los datos se obtuvieron durante el mes de octubre de 2015 mediante el envío del cuestionario por medio de correo electrónico a personal directivo o de nivel estratégico de diferentes áreas funcionales de cada una de las empresas consultadas El tamaño de la muestra, de 102 empresas, permite garantizar una potencia de prueba satisfactoria que es superior al $80 \%$ (Cohen J., 1988).

Tabla 1. Características de las empresas de la muestra

\begin{tabular}{|c|c|c|}
\hline Sector & Frecuencia & Porcentaje \\
\hline $\begin{array}{l}\text { Actividades de administración empresarial; actividades de } \\
\text { consultoría de gestión }\end{array}$ & 23 & $22,55 \%$ \\
\hline Información y comunicaciones & 16 & $15,69 \%$ \\
\hline Educación & 12 & $11,76 \%$ \\
\hline Confección de prendas de vestir & 8 & $7,84 \%$ \\
\hline Actividades de atención de la salud humana y de asistencia social & 6 & $5,88 \%$ \\
\hline Alojamiento y servicios de comida & 6 & $5,88 \%$ \\
\hline $\begin{array}{l}\text { Comercio al por mayor y al por menor; reparación de vehículos } \\
\text { automotores y motocicletas }\end{array}$ & 5 & $4,90 \%$ \\
\hline $\begin{array}{l}\text { Fabricación de productos elaborados de metal, excepto maquinaria } \\
\text { y equipo }\end{array}$ & 4 & $3,92 \%$ \\
\hline Fabricación de sustancias y productos químicos & 4 & $3,92 \%$ \\
\hline Otras actividades Manfactureras & 4 & $3,92 \%$ \\
\hline Transporte y almacenamiento & 3 & $2,94 \%$ \\
\hline Fabricación de aparatos y equipo eléctrico & 2 & $1,96 \%$ \\
\hline $\begin{array}{l}\text { Fabricación de instrumentos, aparatos y materiales médicos y } \\
\text { odontológicos (incluido mobiliario) }\end{array}$ & 2 & $1,96 \%$ \\
\hline Fabricación de juegos, juguetes y rompeCAezas & 1 & $0,98 \%$ \\
\hline Actividades financieras y de seguros & 1 & $0,98 \%$ \\
\hline Actividades inmobiliarias & 1 & $0,98 \%$ \\
\hline $\begin{array}{l}\text { Distribución de agua; evacuación y tratamiento de aguas } \\
\text { residuales, gestión de desechos y actividades }\end{array}$ & 1 & $0,98 \%$ \\
\hline Otras actividades de servicios & 3 & $2,94 \%$ \\
\hline Tamaños por número de empleados & Frecuencia & Porcentaje \\
\hline Grandes Empresas & 25 & $24,51 \%$ \\
\hline Pymes & 77 & $75,49 \%$ \\
\hline Área funcional del encuestado & Frecuencia & Porcentaje \\
\hline Presidencia o Gerencia General & 51 & $50,00 \%$ \\
\hline Sistemas y Tecnología & 14 & $13,73 \%$ \\
\hline Mercadeo & 12 & $11,76 \%$ \\
\hline Otros & 8 & $7,84 \%$ \\
\hline Investigación y Desarrollo & 6 & $5,88 \%$ \\
\hline Finanzas & 5 & $4,90 \%$ \\
\hline Producción & 3 & $2,94 \%$ \\
\hline Recursos Humanos & 3 & $2,94 \%$ \\
\hline
\end{tabular}


El constructo CTI se midió haciendo uso de la escala propuesta por Lu y Ramamurthy, (2011), de donde se tomaron once ítems. Se utiliza una escala de Likert que va desde totalmente de acuerdo (1), hasta totalmente en desacuerdo (5) para los ítems del 1 al 7, y una otra escala Likert que va desde es muy inferior en comparación con otras empresas del sector (1) y es muy superior en comparación con otras empresas del sector (5) para los ítems de 8 al 11. Por su parte, la CA se mide con una escala extraída del trabajo de Flatten et al. (2011), compuesta por catorce ítems que corresponden a la valoración de cada una de las cuatro dimensiones de la CA: Adquisición, Asimilación, Transformación y Explotación, y se utilizó una escala de Likert que va desde totalmente de acuerdo (1), hasta totalmente en desacuerdo (5). Por último, la valoración de las CEB se hizo usando la escala Zhu et al. (2015), que consta de once ítems para la valoración de Compras en línea, Manejo de Canales en línea y servicio al cliente en línea. Para todos los ítems dentro del formato de encuesta fueron medidos con escala Likert de cinco puntos que va desde totalmente de acuerdo (1), hasta totalmente en desacuerdo (5) (Ver Tabla 2).

\section{ANÁLISIS DE RESULTADOS}

El análisis de resultados se presenta en seis subsecciones: varianza del método común, fiabilidad y validez, validez discriminante, variables de control, test del efecto mediador, y predictibilidad y relevancia predictiva.

\section{Varianza del método común}

Para evitar el problema causado por varianza del método común, se tomaron varias medidas relacionadas con el diseño del formulario de encuesta y la toma de los datos. Varias de las acciones tomadas fueron: Redactar las preguntas para evitar inducir sesgo, garantizar el anonimato de las empresas y personas que respondieron el formulario, distribuir las preguntas, en el formulario, de forma que no influyeran alguna respuesta en particular, elegir personal directivo de las empresas, hacer encuestas de prueba y cambiar el título del enCAezado en cada pregunta en la escalas Likert (Chang et al., 2010; Conway y Lance, 2010). La prueba del factor simple de Harman confirma que los datos recolectados tienen la varianza explicada por el primer factor del $43,87 \%$. Lo cual disminuye la posibilidad de que los datos presenten el problema en mención. (Podsakoff et al., 2003).

\section{Fiabilidad y validez}

Para el análisis del modelo de medición reflectivo se ha usado el enfoque de mínimos cuadrados parciales o PLS (Partial Least Squares) para probar el modelo teórico. Específicamente, se usó software SmartPLS 2.0 para este análisis (Henseler et al., 2009). La fiabilidad individual para cada ítem de la escala de medida se verificó siendo cada carga factorial mayor a 0,6. Para investigaciones exploratorias, los valores entre 0.6 y 0,7 son aceptables, mientras que para estados más avanzados de la investigación, los valores entre 0.7 y 0,9 son satisfactorios (Nunnally y Bernstein, 1994). El índice de Varianza Extraída Promedio (AVE) es un medio para evaluar la validez convergente. El valor mínimo recomendado de AVE es de 0,5 (Fornell y Larcker, 1981; Hair et al., 2014). Todos los constructos de este estudio tienen un AVE mayor al mínimo requerido de 0,5 . Del mismo modo, el índice de fiabilidad Compuesta para cada constructo es superior a 0,7 (Chi et al., 2010) (ver Tabla 3).

\section{Validez discriminante}

Para establecer la validez discriminante, se verificó el cumplimiento de dos criterios, en primero de ellos, el Fornell Larcker, en este caso se pudo constatar que la raíz cuadrada de la varianza extraída para cada uno de los constructos es superior a las correlaciones entre cada uno de los distintos constructos (Chin, 1998). En segundo lugar, se confirmó que todos los valores Heterotrait-Monotrait (HTMT) estuvieran por debajo del umbral de 0.85 ó 0.90 (Henseler et al., 2015) (ver Tabla 4).

\section{Variables de control}

Para este estudio, se tomaron cinco variables de control dado que investigaciones previas han demostrado la incidencia de ambas sobre capacidad de absorción y capacidad de e-business (Zhou y Li, 2010; Liu et al., 2013). Por ende, se incorporaron de la siguiente manera: Tamaño: Esta variable corresponde al logaritmo natural de los números de empleados de las empresas; Sector (dummy): 0 representa empresas del sector manufacturero y 1 del sector servicios; Localización geográfica (dummy): 0 empresas fuera de Antioquia y Bogotá y 1 en Antioquia y Bogotá; Antigüedad: es el logaritmo natural de los años de antigüedad de las empresas; Intensidad tecnológica y de conocimiento (dummy): Para el caso de sector manufacturero "0" agrupa empresas de baja y media - baja intensidad de tecnología y el "1" empresas de media alta y alta intensidad de tecnología. Para el caso de sector servicios "0" agrupa empresas de baja intensidad de conocimiento y "1" empresas de alta intensidad de conocimiento (Eurostat, 2009). 
Tabla 2. Escalas de Medida

CTI: Capacidades de las Tecnologías de la Información (Lu \& Ramamurthy, 2011)

*Medido con escala Likert de 5 puntos: 1 significa "Muy inferior en comparación con otras empresas del sector" y 5 significa "Muy superior en comparación con otras empresas del sector"; ${ }^{* *}$ Medido con escala Likert de 5 puntos: 1 significa "Totalmente en desacuerdo" y 5 es "Totalmente de acuerdo".

$\mathrm{CTI1}^{*}$ Los servicios de TI para almacenar, compartir y facilitar el acceso a datos e información (por ejemplo, bases de datos, servicios en la nube), son:

$\mathrm{CTI}^{*} \quad$ Los servicios de TI para las comunicaciones en línea (por ejemplo, videoconferencias, red social corporativa), son:

$\mathrm{CTI}^{*} \quad$ El software y las aplicaciones que apoyan la operación del negocio (por ejemplo, ERP, CRM), son:

$\mathrm{CTI}^{*}$ El hardware y las instalaciones físicas para la prestación de servicios de TI (por ejemplo, datacenter, servidores), son:

CTI5* El desarrollo de una visión clara respecto a cómo las TI agregan valor al negocio, ha sido:

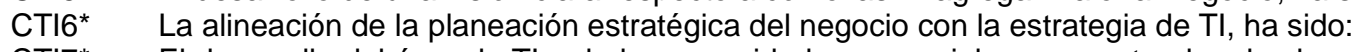

$\mathrm{CTI}^{*} \quad$ El desarrollo del área de TI y de las capacidades gerenciales para entender el valor de las inversiones en $\mathrm{TI}$, ha sido:

$\mathrm{CTI}^{* *} \quad$ Se mantiene actualizada con la información más reciente sobre innovaciones tecnológicas.

CTI9** Tiene la capacidad de experimentar con nuevas Tecnologías de Información (TI) cuando es necesario.

$\mathrm{CTI}^{* *}$ Tiene un clima que apoya y aprueba nuevas formas de utilizar las Tecnologías de Información (TI).

$\mathrm{CTI11}^{* *}$ Busca constantemente nuevas formas de mejorar la eficacia en el uso de Tecnologías de Información (TI).

CEB: Capacidades de E-business (Zhu, Zhao, Tang, \& Zhang, 2015)

*Medido con escala Likert de 5 puntos: 1 es "Totalmente en desacuerdo" y 5 es \#Totalmente de acuerdo".

CEB1* Las operaciones del proceso de compra de suministros en línea han sido reestructuradas para poder facilitar negociaciones y transacciones

CEB2* La programación de las actividades de producción de la empresa se comparte en línea con los proveedores para gestionar los tiempos de compra de suministros.

CEB3* La lista de órdenes de compras se comparte en línea con los proveedores para facilitar la compra de productos.

CEB4* La información sobre las necesidades de materias primas de la empresa se comparte en línea con los proveedores para facilitar la compra de suministros.

CEB5* Se proporciona el servicio al cliente en la página web.

CEB6* Hay servicios de valor agregado en el sitio web para clientes potenciales (por ej. resolución de inquietudes sobre las características y la calidad de los productos y servicios)

CEB7 $^{*} \quad$ Se dispone de un sistema de servicio al cliente en línea para atender las sugerencias y las observaciones de los clientes.

CEB8* El proceso de transacciones en línea ha sido reestructurado para poder soportar la gestión de pedidos de clientes.

CEB9* Las políticas de mercadeo se comparten en línea con los distribuidores para facilitar la promoción de los productos o servicios

CEB10* El catálogo de productos y servicios se comparte en línea con los distribuidores para facilitar la gestión del producto y de los precios.

CEB11* La programación de las actividades de producción de la empresa se comparte en línea con los distribuidores para facilitar el cumplimiento de pedidos de clientes.

CA: Capacidad de Absorción (Zerwas, 2011)

*Medido con escala Likert de 5 puntos: 1 significa "Totalmente en desacuerdo y 5 significa "Totalmente de acuerdo"

\begin{tabular}{|c|c|}
\hline $\mathrm{CA} 1^{*}$ & $\begin{array}{l}\text { La búsqueda de información relevante sobre la industria a la que pertenece la empresa es una tarea de todos } \\
\text { los días. }\end{array}$ \\
\hline $\mathrm{CA} 2^{*}$ & $\begin{array}{l}\text { Los directivos motivan a los empleados a utilizar fuentes de información sobre el sector al que pertenece la } \\
\text { empresa. }\end{array}$ \\
\hline $\mathrm{CA}^{*}$ & $\begin{array}{l}\text { Los directivos esperan que los empleados estén familiarizados con información de otras industrias distintas a } \\
\text { la que pertenece la empresa. }\end{array}$ \\
\hline $\mathrm{CA}^{*}{ }^{*}$ & Se intercambian ideas y conceptos entre las distintas áreas funcionales de la empresa. \\
\hline $\mathrm{CA}^{*}$ & Los directivos fomentan el apoyo entre las distintas áreas de la empresa para resolver problemas. \\
\hline $\mathrm{CA}^{*}$ & $\begin{array}{l}\text { La información fluye rápidamente entre los colaboradores, por ejemplo, si un área adquiere información } \\
\text { importante, la comunica rápidamente a las otras áreas de la empresa. }\end{array}$ \\
\hline $\mathrm{CA} 7^{*}$ & $\begin{array}{l}\text { Los directivos promueven reuniones periódicas entre áreas funcionales para intercambiar nuevos desarrollos, } \\
\text { problemas y logros. }\end{array}$ \\
\hline CA8* & $\begin{array}{l}\text { Los empleados tienen la capacidad de estructurar y utilizar el conocimiento externo que ha adquirido la } \\
\text { empresa. }\end{array}$ \\
\hline CA9* & $\begin{array}{l}\text { Los empleados usualmente absorben nuevo conocimiento y lo organizan para que esté disponible para otros } \\
\text { y pueda ser usado posteriormente }\end{array}$ \\
\hline CA10* & Los empleados articulan de manera exitosa los conocimientos existentes con nuevas ideas y puntos de vista. \\
\hline $\mathrm{CA} 11^{*}$ & Los empleados son capaces de aplicar los nuevos conocimientos en su trabajo. \\
\hline CA12* & Los directivos apoyan el desarrollo de prototipos. \\
\hline CA13* & La empresa regularmente reevalúa tecnologías existentes y las adapta de manera novedosa. \\
\hline CA14* & $\begin{array}{l}\text { La empresa tiene la capacidad de trabajar de manera más efectiva mediante la adopción de nuevas } \\
\text { tecnologías. }\end{array}$ \\
\hline
\end{tabular}


Tabla 3. Fiabilidad y validez convergente. ${ }^{*} p \leq 0.001$

\begin{tabular}{|c|c|c|c|c|c|}
\hline Constructos & Carga Estandarizada & Error Estándar & Valor $\mathrm{t}$ & $\begin{array}{l}\text { Fiabilidad } \\
\text { Compuesta }\end{array}$ & $\begin{array}{l}\text { Índice de } \\
\text { Varianza Extraída }\end{array}$ \\
\hline CTI & & & & 0,95 & 0,64 \\
\hline CTI1 & $0,79^{*}$ & 0,06 & 13,38 & & \\
\hline CTI2 & $0,89^{*}$ & 0,02 & 41,83 & & \\
\hline СТІ3 & $0,89^{*}$ & 0,02 & 49,04 & & \\
\hline CTI4 & $0,78^{*}$ & 0,05 & 15,78 & & \\
\hline CTI5 & $0,79^{*}$ & 0,04 & 20,07 & & \\
\hline CTI6 & $0,78^{*}$ & 0,05 & 15,16 & & \\
\hline CTI7 & $0,78^{*}$ & 0,05 & 16,59 & & \\
\hline СTI8 & $0,79^{*}$ & 0,04 & 18,09 & & \\
\hline CTI9 & $0,77^{*}$ & 0,05 & 16,24 & & \\
\hline CTI10 & $0,79^{*}$ & 0,04 & 17,81 & & \\
\hline CTI11 & $0,71^{*}$ & 0,08 & 9,2 & & \\
\hline CEB & & & & 0,92 & 0,52 \\
\hline CEB1 & $0,66^{*}$ & 0,07 & 8,95 & & \\
\hline CEB2 & $0,78^{*}$ & 0,05 & 16,64 & & \\
\hline CEB3 & $0,75^{\star}$ & 0,05 & 14,6 & & \\
\hline CEB4 & $0,73^{*}$ & 0,06 & 11,42 & & \\
\hline CEB5 & $0,69^{*}$ & 0,07 & 9,77 & & \\
\hline CEB6 & $0,61^{*}$ & 0,1 & 6,07 & & \\
\hline CEB7 & $0,66^{*}$ & 0,09 & 7,81 & & \\
\hline CEB8 & $0,74^{\star}$ & 0,07 & 11,22 & & \\
\hline CEB9 & $0,76^{*}$ & 0,04 & 17,36 & & \\
\hline CEB10 & $0,77^{*}$ & 0,04 & 17,11 & & \\
\hline CEB11 & $0,78^{*}$ & 0,04 & 19,53 & & \\
\hline CA & & & & 0,96 & 0,61 \\
\hline CA1 & $0,76^{\star}$ & 0,05 & 13,9 & & \\
\hline CA2 & $0,81^{*}$ & 0,05 & 16,83 & & \\
\hline CA3 & $0,77^{*}$ & 0,05 & 16,75 & & \\
\hline CA4 & $0,81^{*}$ & 0,04 & 20,81 & & \\
\hline CA5 & $0,82^{*}$ & 0,04 & 21,34 & & \\
\hline CA6 & $0,77^{\star}$ & 0,05 & 15,92 & & \\
\hline CA7 & $0,75^{*}$ & 0,06 & 12,73 & & \\
\hline CA8 & $0,81^{*}$ & 0,05 & 16,77 & & \\
\hline CA9 & $0,75^{*}$ & 0,07 & 11,19 & & \\
\hline CA10 & $0,79^{*}$ & 0,06 & 14,13 & & \\
\hline CA11 & $0,69^{*}$ & 0,06 & 11,52 & & \\
\hline CA12 & $0,77^{*}$ & 0,05 & 15,41 & & \\
\hline CA13 & $0,82^{*}$ & 0,04 & 18,8 & & \\
\hline CA14 & $0,82^{*}$ & 0,04 & 21,57 & & \\
\hline \multicolumn{6}{|l|}{ Variables de Control } \\
\hline Antigüedad & 1 & 0 & 0 & & \\
\hline Región & 1 & 0 & 0 & & \\
\hline Sector & 1 & 0 & 0 & & \\
\hline Tamaño & 1 & 0 & 0 & & \\
\hline $\begin{array}{l}\text { Intensidad tecnológica y } \\
\text { de conocimiento }\end{array}$ & 1 & 0 & 0 & & \\
\hline
\end{tabular}

Tabla 4. Validez discriminante

\begin{tabular}{llccccc}
\hline & & \multicolumn{2}{c}{ Fornell-Larcker } & \multicolumn{2}{c}{ HTMT } \\
\hline \multicolumn{1}{r}{ Constructos } & 1 & 2 & 3 & 1 & 2 \\
\hline 1. & CTI & 0,799 & & & & \\
2. & CEB & 0,562 & 0,724 & & 0,55 & \\
3. & CA & 0,686 & 0,542 & 0,782 & 0,71 & 0,54 \\
\hline
\end{tabular}




\section{Test del efecto mediador}

Para determinar el nivel de significancia estadística de los coeficientes en las relaciones directas y de mediación del modelo usando el método de los mínimos cuadrados (Hair et al., 2014), se ha ejecutado un procedimiento de remuestreo o bootstraping de 5000 submuestras (Henseler et al., 2009) de esta forma se obtienen los valores t y los errores estándar de los coeficientes sobre las trayectorias. Para establecer la mediación, se compararon el modelo directo y mediado para establecer si hay un debilitamiento de las relaciones directas (Baron y Kenny, 1986). Adicionalmente, se efectuó el análisis bootstrap percentil (Williams y MacKinnon, 2008; Preacher y Hayes, 2008), para confirmar de manera concluyente la existencia del efecto mediador de la CA en la relación entre CTI y CEB. Además, e calculó la magnitud de la mediación mediante el test de la varianza explicada o VAF (por su siglas en inglés), que permite establecer si la mediación es parcial o total. (Vinzi et al., 2010).

\section{Predictibilidad y relevancia predictiva.}

Para evaluar la predictibilidad del modelo directo y del modelo mediado se verificó el coeficiente R2 de los constructos endógenos. En todos los casos superó el valor mínimo de 0,1 (Falk y Miller, 1992). En el modelo mediado, $57 \%$ de la varianza de las CEB es explicada, siendo este un nivel de predictibilidad satisfactorio. Así mismo, los valores Q2 para los constructos dependientes son superiores a 0, con esto se evidencia la existencia de la relevancia predictiva de los dos modelos de hipótesis (Hair et., 2014)

\section{RESULTADOS}

Los análisis estadísticos usando las técnicas PLS se efectuaron sobre la estructura de relaciones planteadas en $\mathrm{H} 1$ y H2. Para el modelo directo, en la Tabla 5 se evidencia la influencia de CTI sobre CEB $(\beta=0,58$, valor $t=7,16)$, por ende, se acepta la $\mathrm{H} 1$. Sin embargo, en el modelo mediado, la influencia de CTI sobre CEB ya no es significativa $(\beta=0,23$, valor $t=1,82)$; sumado, a ello, se evidencia que la relación entre CTI y CA es significativa $(\beta=0,78$, valor $t=13,23)$, como también lo es la relación entre CA y CEB $(\beta=0,42$, valor $t=3,43)$. De esta forma se acepta parcialmente la $\mathrm{H} 2$. De otra parte, las variables de control no muestran relaciones de influencia significativa sobre los constructos de ambos modelos.

Tabla 5. Resultados de las ecuaciones estructurales. * $p<0.001$

\begin{tabular}{|c|c|c|c|}
\hline Modelo & Trayectorias & Coeficiente (Beta) & Valor $\mathrm{t}$ \\
\hline \multirow{7}{*}{ Directo } & $\begin{array}{l}\mathrm{CTI} \rightarrow \mathrm{CEB} \\
\left(\mathrm{R}^{2}=0,38 ; \mathrm{Q}^{2}=0,61\right)\end{array}$ & $0,58^{*}$ & 7,16 \\
\hline & \multicolumn{3}{|c|}{ Variables de Control } \\
\hline & Antigüedad $\rightarrow$ CEB & 0,1 & 0,82 \\
\hline & Localización geográfica $\rightarrow$ CEB & $-0,01$ & 0,1 \\
\hline & Sector $\rightarrow$ CEB & $-0,1$ & 1,15 \\
\hline & Tamaño $\rightarrow$ CEB & 0,02 & 0,18 \\
\hline & Intensidad tecnológica y de conocimiento $\rightarrow$ CEB & $-0,09$ & 1,08 \\
\hline \multirow{14}{*}{ Mediado } & $\mathrm{CTI} \rightarrow \mathrm{CEB}$ & 0,23 & 1,82 \\
\hline & $\begin{array}{l}\mathrm{CTI} \rightarrow \mathrm{CA} \\
\left(\mathrm{R}^{2}=0,44 ; \mathrm{Q}^{2}=0,54\right)\end{array}$ & $0,78^{*}$ & 13,23 \\
\hline & $\begin{array}{l}C A \rightarrow C E B \\
\left(R^{2}=0,57 ; Q^{2}=0,52\right) ;\end{array}$ & $0,42^{*}$ & 3,43 \\
\hline & \multicolumn{3}{|c|}{ Variables de Control } \\
\hline & Antigüedad $\rightarrow$ CEB & 0,13 & 1,09 \\
\hline & Localización geográfica $\rightarrow$ CEB & 0,04 & 0,41 \\
\hline & Sector $\rightarrow$ CEB & $-0,06$ & 0,72 \\
\hline & Tamaño $\rightarrow$ CEB & 0,14 & 1,24 \\
\hline & Intensidad tecnológica y de conocimiento $\rightarrow$ CEB & $-0,1$ & 1,18 \\
\hline & Antigüedad $\rightarrow \mathrm{CA}$ & $-0,07$ & 0,81 \\
\hline & Localización geográfica $\rightarrow$ CA & $-0,1$ & 1,68 \\
\hline & Sector $\rightarrow$ CA & $-0,1$ & 1,07 \\
\hline & Tamaño $\rightarrow$ CA & $-0,27$ & 3 \\
\hline & Intensidad tecnológica y de conocimiento $\rightarrow \mathrm{CA}$ & 0,01 & 0,09 \\
\hline
\end{tabular}

La prueba del bootstrap-percentil que genera un intervalo de confianza del 95\% e implica el cálculo del producto de los efectos indirectos con los datos del remuestreo, indica que el intervalo establecido no 
contiene el valor cero (ver tabla 6), lo que implica que el efecto mediador de CA es significativo (Williams y MacKinnon, 2008; Preacher y Hayes, 2008). Sumado a ello, el VAF es de 58.9\%, lo cual indica que la mediación es parcial (Vinzi et al., 2010).

Tabla 6. Test del efecto mediador

\begin{tabular}{ccccccc}
\hline \multicolumn{2}{c}{ Efecto Total } & \multicolumn{2}{c}{ Efecto Directo } & $\begin{array}{c}\text { Efecto Indirecto } \\
\left(\mathrm{a}^{*} \mathrm{~b}\right)\end{array}$ & \multicolumn{2}{c}{$\begin{array}{c}\text { Bootstrap Percenti//ntervalo de } \\
\text { confianza (95\%) }\end{array}$} \\
\hline Coeficiente & Valor $\mathrm{t}$ & Coeficiente & Valor t & Coeficiente & Límite inferior & $\begin{array}{c}\text { Límite } \\
\text { superior }\end{array}$ \\
\hline 0,58 & 7,16 & 0,23 & 1,82 & 0,33 & 0,13 & 0,54 \\
\hline
\end{tabular}

En suma, con base en los resultados del test bootstrap-percentil y del VAF (ver tabla 6), se aceptan la H1 y la H2. Por ende, existe una relación positiva entre CTI y CEB, así como un efecto mediador de la CA sobre la dicha relación. En particular el VAF de $58.9 \%$, indica que la influencia de CTI puede no ser suficiente para un desempeño aceptable de las CEB y que es necesaria la influencia de los procesos de CA para lograr mejores efectos en el desarrollo de los procesos de tipo organizacional, la definición de estrategias y la lectura del entorno de la compañía que son aplicados a las CEB. No obstante, aun cuando en el modelo mediador se nota una gran influencia de las CA, las CTI aún tienen incidencia en la relación con CEB, puesto que la madurez de la base tecnología, la articulación de las TI en la estrategia empresarial y la vigilancia tecnológica aplicada a las TI son factores esenciales para el fortalecimiento de las plataformas de e-business.

No obstante, el modelo revela que la incidencia mediadora de CA es pieza clave para poder observar el entorno y aplicar el conocimiento de diferentes fuentes aplicado a las CEB con el fin de impactar positivamente canales, clientes, actividades de tipo organizacional y procesos relacionados con proveedores que usan los servicios electrónicos de las empresas. Si no se toma en cuenta lo anterior, los efectos de CTI sobre el desempeño de CEB, pueden ofrecer plataformas robustas tecnológicamente hablando, pero definitivamente carentes de resultados óptimos, porque en cierto modo se está ignorando la información disponible en cuanto al entorno organizacional, perspectivas, tendencias y necesidades que pueden ser fundamentales al momento de crear escenarios de tipo prospectivo, el diseño de estrategias, desarrollo de nuevos productos y procesos, entre otras actividades que son inherentes al desarrollo de las plataformas de e-business.

\section{DISCUSIÓN FINAL}

Las implicaciones teóricas del estudio están acordes con investigaciones previas que han analizado la mediación parcial de CA sobre la relación de CTI sobre otros constructos (Tanriverdi, 2005; Francalanci y Morabito, 2008; Datta, 2012; Liu et al., 2013; Tzokas et al., 2015; Roldán et al., 2015). Es así como esta investigación ha afianzado la teoría existente relacionada con la forma en la que la CA ejerce su complementariedad en las diferentes relaciones que tienen las CTI con varios constructos organizacionales, como la eficiencia organizacional, la eficiencia financiera y la habilidad de comercializar innovaciones, todo ello de forma parcial, es decir, sin la desaparición de los efectos directos. Adicionalmente, el modelo de mediación planteado no se ha estudiado en investigaciones previas por ende este estudio aporta nueva evidencia en ese sentido.

El modelo de mediación demostrado, ha dado mayor protagonismo a la CA en el entorno de dos constructos que se han caracterizado por el ambiente técnico, evidenciando la necesidad de articular la parte más instrumental con diferentes capacidades, sobre todo de carácter blando como CA, en contraste con los constructos duros, basados en la tecnología. De esta forma, en este estudio aporta a la discusión relacionada con el valor real que las TI generan en las empresas, demostrando que es necesario articular a las CTI, conocimientos de diversas fuentes y disciplinas para explotar todo su potencial. Es así como, los hallazgos de esta investigación proponen que se combinen las CTI con las rutinas propias de la CA para poder tener un retorno más alto, sobre todo de los resultados que se obtienen de la explotación de CEB.

En ese mismo sentido, el aporte que se genera a la gestión en las empresas, radica en el enfoque que se debe dar a las nuevas iniciativas de e-bussines, que tienen que estar ligadas a la influencia de otras fuentes de conocimiento más allá de lo netamente técnico y que puede ser articulado con todas las dependencias de la empresa, fortalecer sinergias y desarrollar, productos, servicios y plataformas valoradas por el mercado. De esta forma el desarrollo de las CTI y las prácticas de los procesos de CA producirán recursos escasos y difíciles de imitar, fuente de ventajas competitivas. 
De otra parte, el estudio ha tenido algunas limitaciones, que se han revelado de mano del análisis de fiabilidad y validez convergente, que ha arrojado resultados aceptables, pero no óptimos sobre algunos ítems en la escala de medida de las CEB (Nunnally y Bernstein, 1994; Chin, 1998; Hair et al., 2014). Esta limitación es válida como fuente de futuras investigaciones que permitan mejorar o construir las escalas de medición y llevarlas a su madurez máxima, y así medir las CEB y sus demás constructos constitutivos con elementos de más fiabilidad. Adicionalmente, se puede extender el ámbito de estudios de las influencias de CTI con capacidades relacionadas con E-business, como e-learning, m-business o negocios móviles, teletrabajo, o incluso telemedicina y telesalud, puesto que estas aplicaciones tecnológicas tienen raíces comunes y podrían ayudar a enriquecer el conocimiento que se tiene de las dinámicas de CEB.

Reforzando lo anterior, es importante considerar que la tendencia vista en estudios previos, apunta a realizar investigaciones donde se hace uso de capacidades organizacionales de carácter interno como mediadoras en las relaciones entre constructos similares al objeto de estudio de esta investigación. En ese sentido, es recomendable establecer la mediación de Capacidades externas sobre la relación entre CTI y CEB, y así romper ese cerco que sólo considera capacidades internas. Una alternativa sería el estudio de la capacidad de la innovación abierta, que implica elementos de colaboración hacia afuera de las empresas.

\section{CONCLUSIONES}

De acuerdo a lo presentado y discutido en este trabajo, se puede extraer las siguientes conclusiones principales: (1) las implicaciones teóricas del estudio están acordes con investigaciones previas que han analizado la mediación parcial de CA sobre la relación de CTI sobre otros constructos; y (2) el modelo de mediación demostrado, ha dado mayor protagonismo a la CA en el entorno de dos constructos que se han caracterizado por el ambiente técnico

\section{REFERENCIAS}

Addas, S., y A. Pinsonneault, IT capabilities and NPD performance: examining the mediating role of team knowledge processes, Knowledge Management Research and Practice: 14(1), 1-20 (2014)

Alderete, M., y L. Gutiérrez, TIC y productividad en las industrias de servicios en Colombia. Lecturas de Economía: (77),163-188 (2012)

Armstrong, G. y P. Kotler, Marketing: An introduction, $7^{a}$ edición. Prentice Hall, New Jersey, USA (2005).

Baron, R.M., y D.A. Kenny, The moderator-mediator variable distinction in social psychological research: Conceptual, strategic, and statistical considerations: Journal of Personality and Social Psychology, 51(6), 1173-1182 (1986)

Barney, J. y Ray, G, How information technology resources can provide a competitive advantage in customer service, Planning for Information Systems, 3(2), 444-460 (2015)

Barua, A.; P. Konana y otros dos autores, An empirical investigation of net-enabled business value: Mis Quarterly, 28(4), 585-620 (2004)

Bharadwaj, A.S., A resource-based perspective on information technology capability and firm performance: an empirical investigation: MIS Quarterly, 169-196 (2000)

Cano, J.A. y J.J Baena, Tendencias en el uso de las tecnologías de información y comunicación para la negociación internacional: Estudios Gerenciales, 31, 335-346 (2015)

Carr, N.G., IT Doesn't Matter: Harvard Business, 81(5), 41 - 59 (2003)

Cegarra, J.G.; D.J. Jiménez y E.A. Martínez, Implementing e-business through organizational learning: An empirical investigation in SMEs: International Journal of Information Management, 173-186 (2007)

Chang, H.; Y.C. Tsai y C.H. Hsu, E-procurement and supply chain performance: Supply Chain Management: An International Journal, 18(1), 34-51 (2013)

Chang, K.H., y D.F. Gotcher, Conflict-coordination learning in marketing channel relationships: The distributor view: Industrial Marketing Management, 39(2), 287-297 (2010)

Chang, S.J.; A.V. Witteloostuijn y L. Eden, From the Editors: Common method variance in international business research: Journal of International Business Studies, 41, 178-184 (2010)

Chen, J.S. y R.K. Ching, An empirical study of the relatinship of IT intensity and organizational absorptive capacity on CRM performance: Advanced Topics in Global Information Management (4), 317-334. (2005) 
Chen, Y.; Y. Wang y otros 4 autores, IT capability and organizational performance: the roles of business process agility and environmental factors: European Journal of Information Systems, $326-342$ (2014)

Chi, M.; J. Zhao y otros dos autores, Analysis of E-business Capabilities and Performance: From e-SCM Process View: Proceedings - 2010 3rd IEEE International Conference on Computer Science and Information Technology, 18-22 (2010)

Chin, W.W., The partial least squares approach to structural equation modeling: Modern methods for business research, 295-336 (1998)

Choi, Y., The Drives of E-Marketplace Adoption in China: The Mediation Effect of Trust: 2012 International Conference on Management of e-Commerce and e-Government (ICMeCG), 253 - 257 (2012)

Cohen, J., Statistical Power Analysis for the Behavioral Sciences: 2a edición, New Jerey, USA (1988)

Cohen, W.M., y D.A. Levinthal, Absorptive Capacity: A New Perspective on Learning and Innovation: Administrative Science Quarterly, 35 (1), 128-152 (1990)

Conway, J.M., y C.E. Lance, What Reviewers Should Expect from Authors Regarding Common Method Bias in Organizational Research: Journal of Business and Psychology, 25 (3), 325-334 (2010)

Croom, S., The impact of web-based procurement on the management of operating resources supply: The Journal of Supply Chain Management, 4-13 (2000)

DANE, Indicadores básicos de tenencia y uso de las tecnologías de la información y la comunicación en empresas (en la web http://www.dane.gov.co/files/investigaciones/boletines/tic/bol_empresas_2014def.pdf, acceso 25 de enero 2016), Bogotá (2015)

Datta, A., IT-Based Knowledge Capability and Commercialization of Innovations: Modeling the Impacts of Ambidexterity and Absorptive Capacity: International Journal of Knowledge Management, 84-98 (2012)

Davila, A.; M. Gupta y R. Palmer, Moving Procurement Systems to the Internet:: the Adoption and Use of EProcurement Technology Models: European Management Journal, 21 (1), 11-23 (2003)

Dimitriadis, N. y S. Koh, Information flow and supply chain management in local production networks: the role of people and information systems: Production Planning and Control, 16 (6), 545-554 (2005)

Eikebrokk, T. y D. Olsen, An empirical investigation of competency factors affecting e-business success in European SMEs: Information y Management, 44 (4), 364-383 (2007)

Eng, T.Y., E-customer service capability and value creation: The Service Industries Journal, 28 (9), 12931306 (2008)

Ericsson Consumer LAB, M-Commerce in Latin America (en la web https://www.ericsson.com/res/docs/2013/consumerlab/m-commerce-in-latam.pdf acceso 25 de enero 2016). Estocolmo, Suecia (2013)

Eurostat, 'High-technology' and 'knowledge based services' aggregations based on NACE Rev. 2. (en la web http://ec.europa.eu/eurostat/cache/metadata/Annexes/htec_esms_an3.pdf acceso 25 enero 2016) (2009)

Falk, R.F. y N. B. Miller, A primer for soft modelling: 1a edición, Ohio, USA (1992)

FEC, The Global Information Technology Report 2015 (en la web http://www3. weforum.org/docs/WEF_Global_IT_Report_2015.pdf visto: 25 de enero 2016). Ginebra, Suiza (2015)

Flatten, T.C.; A. Engelena, y otros dos autores, A measure of absorptive capacity: Scale development and validation: European Management Journal, 29(2), 98-116 (2011)

Fornell, C. y D.F. Larcker, Evaluating Structural Equation Models with Unobservable Variables and Measurement Error: Journal of Marketing Research, 18(1), 39-50 (1981)

Francalanci, C. y V. Morabito, IS integration and business performance: The mediation effect of organizational absorptive capacity in SMEs: Journal of Information Technology, 297-312 (2008)

Gálvez, E., Tecnologías de información y comunicación, e innovación en las MIPYMES de Colombia: Cuadernos de Administración, 71-79 (2014)

Hair, J.F.; G.M. Hult y otros dos autores: A primer on partial least squares structural equation modeling (PLS-SEM): 1a edición, California, USA (2014) 
Henseler, J.; C.M. Ringle y R.R. Sinkovics, The Use of Partial Least Squares Path Modeling in International Marketing: Advances in International Marketing, 20, 277-320 (2009)

Henseler, J.; Ringle, C. M. y Sarstedt, M, A new criterion for assessing discriminant validity in variancebased structural equation modeling. Journal of the Academy of Marketing Science, 43(1), 115-135 (2015)

Hung, S. Y.; Chen, C. C. y Huang, N. H, An Integrative approach to understanding customer satisfaction with e-service of online stores. Journal of Electronic Commerce Research, 15(1), 40-57 (2014)

Janita, I. y W.K. Chong, Barriers of B2B e-Business Adoption in Indonesian SMEs: A Literature Analysis: Procedia Computer Science, 17, 571-578 (2013)

Jiang, Y. y J. Zhao, An empirical research of the forming process of firm inter-organizational e-business capability: based on the supply chain processes: 2010 2nd International Conference on Information Science and Engineering (ICISE), 2603-2606 (2010)

Jiao, H.; I.C. Chang y Y. Lu, The Relationship on Information Technology Capability and Performance: An Empirical Research in the Context of China's Yangtze River Delta Region: IEEM: 2008 International Conference on Industrial Engineering and Engineering Management, 872-876 (2008)

Jiménez, M.M.; V.J. García y L.M. Molina, Validation of an instrument to measure absorptive capacity: Technovation, 190-202 (2011)

Johnson, M.E. y S. Whang, e-Business and supply chain management: an overview and framework: Production and Operations Management, 11(4), 413-423 (2002)

Kaefer, F. y E. Bendoly, Measuring the impact of organizational constraints on the success of business-tobusiness e-commerce efforts: a transactional focus: Information y Management, 41(5), 529-541 (2004)

Koellinger, P., The relationship between technology, innovation, and firm performance-Empirical evidence from e-business in Europe: Research Policy, 1317-1328 (2008)

Lane, P.J. y M. Lubatkin, Relative absorptive capacity and interorganizational learning: Strategic management journal, 19(5), 461-477 (1998)

Lee, C.P.; G.G. Lee y H.F. Lin, The role of organizational capabilities in successful e-business implementation: Business Process Management Journal, 13(5), 677 - 693 (2007)

Lee, M.C. y T. Chang, Linking knowledge management and innovation management in e-business: International Journal Innovation and Learning, 4(2), 145-159 (2007)

Lin, H.F. y G.G. Lee, Impact of organizational learning and knowledge management factors on e-business: Management Decision, 171-188 (2005)

López, D.; Marulanda, C. E., y López, M, Métricas de Valoración de la Gestión del Conocimiento para las Pequeñas y Medianas Empresas del Sector Tecnologías de Información en el Triángulo del Café en Colombia, Información tecnológica, 26(3), 173-183 (2015)

Liu, H.; W. Ke y otros dos autores, The impact of IT capabilities on firm performance: The mediating roles of absorptive capacity and supply chain agility: Decision Support Systems, 1452-1462 (2013)

Lu, Y. y K.R. Ramamurthy, Understanding The Link Between Information Technology Capability And Organizational Agility Malhotra, A.; S. Gosain y O.A. Sawy, Absorptive Capacity Configurations in Supply Chains: Gearing for Partner-Enabled Market Knowledge Creation. MIS Quarterly, 29(1), 145-187 (2005)

Marulanda, C. E.; Giraldo, J. A. y López, M, Evaluación de la Gestión del Conocimiento en las Organizaciones de la Red de Tecnologías de Información y Comunicaciones del Eje Cafetero en Colombia. Información tecnológica, 24(4), 105-116 (2013)

Maditinos, D.; Chatzoudes, D. y Sarigiannidis, L, Factors affecting e-business successful implementation. International Journal of Commerce and Management, 24(4), 300-320 (2014)

Menascé, D.A., Scaling for E-Business: Analysis and Simulation of Computer and Telecommunication Systems, 511-513 (2000)

MinTIC., Panorama TIC (en la web http://colombiatic.mintic.gov.co/602/articles-8917_panoranatic.pdf, acceso: 25 de enero 2016). Bogotá, Colombia (2015) 
Mishra, A.N.; P. Konana y A. Barua, Antecedents and Consequences of Internet Use in Procurement: An Empirical Investigation of U.S. Manufacturing Firms: Information Systems Research, 18(1), 103 - 120 (2007)

Nakata, C.; Z. Zhu y M.L. Kraimer, The Complex Contribution of Information Technology Capability to Business Performance: Journal of Managerial Issues, 20(4), 485-506 (2008)

Ndiege, J.R.; M.E. Herselman y S.V. Flowerday, Absorptive Capacity and ICT Adoption Strategies for SMEs: a Case Study in Kenya: The African Journal of Information Systems, 6(4), 140 - 155 (2014)

Nunnally, J.C. y I. Bernstein, Psychometric Theory. 3a edición, Nueva York, USA (1994)

Oh, L.B.; H.H. Teo y V. Sambamurthy, The effects of retail channel integration through the use of information technologies on firm performance: Journal of Operations Management, 30(5), 368-381 (2008)

Oh, S.; H. Yang y S.W. Kim, Managerial capabilities of information technology and firm performance: role of e-procurement system type: International Journal of Production Research, 52(15), 1-19 (2014)

Paksoy, T.; N.Y. Pehlivan y C. Kahraman, Organizational strategy development in distribution channel management using fuzzy AHP and hierarchical fuzzy TOPSIS: Expert Systems with Applications, 39(3), $2822-2841(2012)$

Patterson, W. y Ambrosini, V. Configuring absorptive capacity as a key process for research intensive firms. Technovation, 36, 77-89 (2015)

Podsakoff, P.M.; S.B. MacKenzie y otros dos autores, Common method biases in behavioral research: $A$ critical review of the literature and recommended remedies: J. Applied Psychology, 88(5), 879 - 903 (2003)

Poon, P. y C. Wagner, Critical success factors revisited: success and failure cased of information systems for senior executives: Decision Support Systems, 30(4), 393 - 418 (2001)

Preacher, K.J. y A.F. Hayes, Asymptotic and resampling strategies for assessing and comparing indirect effects in multiple mediator models: Behavior Research Methods, 40(3), 879 - 891 (2008)

Ray, G.; W.A. Muhanna y J.B. Barney, Information Technology and the Performance of the Customer Service Process: A Resource-Based Analysi: MIS Quarterly, 29(4), 625-652 (2005)

Raymond, L. y F. Bergeron, Enabling the business strategy of SMEs through e-business capabilities- $A$ strategic alignment perspective: Industrial Management y Data Systems, 108(5), 577-595 (2008)

Raymond, L.; F. Bergeron y otros dos autores, Developing Absorptive Capacity through e-Business: The Case of International SMEs: Journal of Small Business Management, 53(S1), 75-94 (2015)

Raymond, L., F. Bergeron y otros dos autores, IT-enabled Knowledge Management for the Competitive Performance of Manufacturing SMEs: An Absorptive Capacity-based View. Knowledge and Process Management, 23(2), 110-123 (2016)

Roche, J., Are you ready for e-procurement?: Strategic Finance, 56-59 (2001)

Roldán, J.L.; A.L. Leal y C. Felipe, Information systems capabilities and organizational agility: Understanding the mediating role of absorptive capacity when influenced by a hierarchy culture: 2nd International Symposium on Partial Least Squares Path Modeling (2015)

Sáenz, M.J.; E. Revilla, y D. Knoppen, Absorptive capacity in buyer-supplier relationships: empirical evidence of its mediating role: Journal of Supply Chain Management, 50(2), 18-40 (2014)

Sambamurthy, V. y R.W. Zmund, At the Heart of Success: Organizationwide Management Competencies. En Steps to the Future: Fresh Thinking on the Management of IT-Based Organizational Transformation por C. Saue, y P.W. Yetton, pp 143 - 164, San Francisco, USA (1997)

Sanders, N.R., An empirical study of the impact of e-business technologies on organizational collaboration and performance: Journal of Operations Management, 25(6), 1332 - 1347 (2007)

Saraf, N.; C.S. Langdon y S. Gosain, IS Application Capabilities and Relational Value in Interfirm Partnerships: Information Systems Research, 18(3), 320-339 (2007)

Shakir, M.; G. Smith y E. Gulee, E-procurement: reaching out to small and medium businesses. MIS Quarterly Executive, 6(4), 225 - 238 (2007)

Shi, X. y Z. Liao, Inter-firm dependence, inter-firm trust, and operational performance: The mediating effect of e-business integration: Information y Management, 52(8), 943 - 950 (2015) 
Singh, A. y Teng, J. T, Enhancing supply chain outcomes through Information Technology and Trust. Computers in Human Behavior, 54, 290-300 (2016)

Srivastava, M.K.; D.R. Gnyawali y D.E. Hatfield, Behavioral implications of absorptive capacity: The role of technological effort and technological capability in leveraging alliance network technological resources: Technological Forecasting and Social Change, 92, 346-358 (2015)

Tanriverdi, H., Information Technology Relatedness, Knowledge Management Capability, and Performance of Multibusiness Firms: MIS Quarterly, 311-334 (2005)

Tzokas, N.; Y.A. Kim y otros dos autores, Absorptive capacity and performance: The role of customer relationship and technological capabilities in high-tech SMEs: Ind. Marketing Manage., 47, 134-142 (2015)

UIT, Informe sobre la Medición de la Sociedad de la Información (en la web https://www.itu.int/en/ITU-D/ Statistics/Documents/publications/misr2015/MISR2015-ES-S.pdf acceso: 25/01/2016). Ginebra, Suiza (2014)

Vinzi, V.E.; L. Trinchera y S. Amato, PLS Path Modeling: From Foundations to Recent Developments and Open Issues for Model Assessment and Improvement En Handbook of Partial Least Squares por V.E. Vinzi, W.W. Chin y otros dos autores, pp $47-82$, Berlín, Alemania (2010)

Weill, P. y M. Vitale, What IT Infrastructure Capabilities are Needed to Implement E-Business Models?: MIS Quarterly Executive, 1(1), 17-34 (2002)

Williams, J. y D. MacKinnon, Resampling and distribution of the product methods for testing indirect effects in complex models: Structural Equation Modeling, 15(1), 23-51 (2008)

Williams, Z. y R. Moore, Supply chain relationships and information capabilities. The creation and use of information power: International J. Physical Distribution y Logistics Management, 37(6), 469 - 483 (2007)

Wu, J.N.; W.J. Zhong y S.E. Mei, Application capability of e-business, e-business success, and organizational performance: Empirical evidence from China: Technol. Forecasting \& Social Change, 1412-1425 (2011)

Yeh, C.H.; G.G. Lee y J.C. Pai, Using a technology-organization environment framework to investigate the factors influencing e-business information technology capabilities. Information Development, 1 - 16 (2014)

Zahra, S.A. y G. George, Absorptive capacity: a review, reconceptualization, and extension: Academy of Management Review, 185 - 203 (2002)

Zerwas, D., Organizational Culture and Absorptive Capacity: 1ª edición, Berlín, Alemania (2011).

Zhao, J.; W.V. Huang y Z. Zhu, An Empirical Study of E-Business Implementation Process in China: IEEE transactions on engineering management, 55(1), 134-147 (2008)

Zhou, K. y C. Li, How strategic orientations influence the building of dynamic capability in emerging economies: Journal of Business Research, 63(3), 224 - 231 (2010)

Zhu, K., The complementarity of information technology infrastructure and e-commerce capability: $A$ resource-based assessment of their business value: Journal of Management Information Systems, 21(1), $167-202(2004)$

Zhu, K., y K.L. Kraemer, Post-Adoption Variations in Usage and Value of E-Business by Organizations: Cross-Country Evidence from the Retail Industry: Information Systems Research, 16(1), 61 - 84 (2005)

Zhu, K.; K.L. Kraemer y J. Dedrick, Information Technology Payoff in E-Business Environments: An International Perspective on Value Creation of E-Business in the Financial Services Industry: Journal of Management Information Systems, 21(1), 17 - 54 (2004)

Zhu, Z.; J. Zhao; X. Tang y Y. Zhang, Leveraging e-business process for business value: A layered structure perspective: Information y Management, 679 - 691 (2015) 